\title{
Chemistry of the Posphinidene Oxide Ligand
}

\author{
María Alonso, M. Esther García, Miguel A. Ruiz*, Hayrullo Hamidov, ${ }^{\dagger}$ and John C. Jeffery. ${ }^{\dagger}$ \\ Departamento de Química Orgánica e Inorgánica/IUQOEM, Universidad de Oviedo, 33071 Oviedo, Spain. ${ }^{\dagger}$ School of \\ Chemistry, University of Bristol, Bristol BS8 ITS, UK.
}

\section{Supporting Information}

Preparative Procedures and Microanalytical Data for New Compounds. $\left(R^{*}=2,4,6-C_{6} \mathrm{H}_{2}{ }^{\mathrm{t}} \mathrm{Bu}_{3}\right)$

\section{General Procedures and Starting Materials}

All manipulations and reactions were carried out under a nitrogen (99.9995\%) atmosphere using standard Schlenk techniques. Solvents were purified according to literature procedures, ${ }^{1}$ and distilled prior to use. Petroleum ether refers to that fraction distilling in the range 65-70 ${ }^{\circ} \mathrm{C}$. Compound $\left[\mathrm{Mo}_{2} \mathrm{Cp}_{2}(\mu-\mathrm{H})\left(\mu-\mathrm{PHR}^{*}\right)(\mathrm{CO})_{4}\right]\left(\mathrm{Cp}=\eta^{5}-\mathrm{C}_{5} \mathrm{H}_{5} ; \mathrm{R}^{*}=\right.$ $\left.2,4,6-\mathrm{C}_{6} \mathrm{H}_{2}{ }^{\mathrm{t}} \mathrm{Bu}_{3}\right)$ was prepared as described previously. ${ }^{2}$ All other reagents were obtained from the usual commercial suppliers and used as received. Chromatographic separations were carried out using jacketed columns cooled by tap water (ca. $15{ }^{\circ} \mathrm{C}$ ). Commercial aluminium oxide (activity I, 150 mesh) was degassed under vacuum prior to use. The latter was mixed under nitrogen with the appropriate amount of water to reach the activity desired. Filtrations were performed using diatomaceous earth. IR stretching frequencies of $\mathrm{CO}$ ligands were measured in solution and are referred to as $v_{\mathrm{CO}}$ (solvent). Nuclear Magnetic Resonance (NMR) spectra were routinely recorded at $300.13\left({ }^{1} \mathrm{H}\right), 121.50\left({ }^{31} \mathrm{P}\left\{{ }^{1} \mathrm{H}\right\}\right)$ or $75.47 \mathrm{MHz}\left({ }^{13} \mathrm{C}\left\{{ }^{1} \mathrm{H}\right\}\right)$ at $290 \mathrm{~K}$ in $\mathrm{CD}_{2} \mathrm{Cl}_{2}$ solutions unless otherwise stated. Chemical shifts $(\delta)$ are given in ppm, relative to internal tetramethylsilane (TMS) or external $85 \%$ aqueous $\mathrm{H}_{3} \mathrm{PO}_{4}$ solutions $\left({ }^{31} \mathrm{P}\right)$. Coupling constants $(J)$ are given in Hertz. Assignments of the ${ }^{13} \mathrm{C}$ NMR resonances for the aryl group follow the labelling shown in the figure below, and are reported as ${ }^{\mathrm{n}} \mathrm{C}\left(\mathrm{C}_{6} \mathrm{H}_{2}\right)$ or ${ }^{\mathrm{m}} \mathrm{C}\left({ }^{t} \mathrm{Bu}\right)$ as required $(\mathrm{n}=1$ to $6 ; \mathrm{m}=1,2)$.

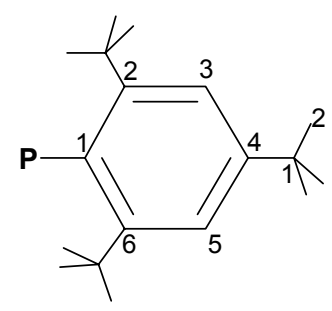

Preparative procedures, spectroscopic and microanalytical data for the new compounds.

Preparation of (H-DBU)[MoCp $\left.\left\{\mathbf{P}(\mathbf{O}) \mathbf{R}^{*}\right\}(\mathbf{C O})_{2}\right]$ (1). Neat 1,8 diazabicyclo [5.4.0] undec-7-ene (DBU, $95 \mu \mathrm{L}, 0.632 \mathrm{mmol}$ ) was added to a dichloromethane solution $(30 \mathrm{~mL})$ of $\left[\mathrm{Mo}_{2} \mathrm{Cp}_{2}(\mu-\mathrm{H})(\mu-\right.$ $\left.\left.\mathrm{PHR}^{*}\right)(\mathrm{CO})_{4}\right](0.300 \mathrm{~g}, 0.421 \mathrm{mmol})$ and the mixture was stirred at $0{ }^{\circ} \mathrm{C}$ for $2 \mathrm{~h}$ to give a dark green solution of the salt (H-DBU) $\left[\mathrm{Mo}_{2} \mathrm{Cp}_{2}(\mu-\right.$ PHR $\left.^{*}(\mathrm{CO})_{4}\right]$. Air $(48 \mathrm{~mL})$ was then injected to the solution using a syringe, the Schlenk tube was closed and the mixture was stirred at room temperature for $1 \mathrm{~h}$ to give an orange solution. The solvent was then removed under vacuum, the residue was extracted with diethylether, and the extracts were filtered. The solvent was then removed from the filtrate, and the residue was crystallized by slow diffusion of layers of diethyl ether and petroleum ether into a concentrated solution of the crude material in toluene. This yielded compound 1 as yellow crystals $(0.162 \mathrm{~g}, 58 \%)$. Anal. Calcd for $\mathrm{C}_{34} \mathrm{H}_{51} \mathrm{MoN}_{2} \mathrm{O}_{3} \mathrm{P}: \mathrm{C}, 61.62 ; \mathrm{H}, 7.76, \mathrm{~N}, 4.23$. Found: $\mathrm{C}, 61.51 ; \mathrm{H}, 7.77$, $\mathrm{N}, 4.25 . v_{\mathrm{CO}}\left(\mathrm{CH}_{2} \mathrm{Cl}_{2}\right): 1874$ (vs), $1790(\mathrm{~s}) \mathrm{cm}^{-1} .{ }^{1} \mathrm{H}$ NMR $(200.13 \mathrm{MHz}$, $\left.\mathrm{C}_{6} \mathrm{D}_{6}\right): \delta 13.88(\mathrm{~s}, 1 \mathrm{H}, \mathrm{NH}), 7.52\left(\mathrm{~s}, 2 \mathrm{H}, \mathrm{C}_{6} \mathrm{H}_{2}\right), 5.11(\mathrm{~s}, 5 \mathrm{H}, \mathrm{Cp}), 3.31$, $2.72,2.15,1.99,1.51,1.12,1.02,0.70\left(8 \times \mathrm{m}, 8 \times 2 \mathrm{H}, \mathrm{CH}_{2}\right), 2.03(\mathrm{~s}$, $\left.18 \mathrm{H}, \mathrm{CH}_{3}\right), 1.37\left(\mathrm{~s}, 9 \mathrm{H}, \mathrm{CH}_{3}\right) .{ }^{31} \mathrm{P}\left\{{ }^{1} \mathrm{H}\right\}$ NMR $(81.04 \mathrm{MHz}): \delta 385(\mathrm{~s})$. ${ }^{13} \mathrm{C}\left\{{ }^{1} \mathrm{H}\right\}$ NMR (100.63 MHz): $\delta 240.8\left(\mathrm{~d}, J_{\mathrm{CP}}=21, \mathrm{CO}\right), 166.2(\mathrm{~s}, \mathrm{CN})$, $155.9\left[\mathrm{~d}, J_{\mathrm{CP}}=30, \mathrm{C}^{1}(\mathrm{Ar})\right], 148.4\left[\mathrm{~s}, \mathrm{C}^{4}(\mathrm{Ar})\right], 146.4\left[\mathrm{~d}, J_{\mathrm{CP}}=6, \mathrm{C}^{2}(\mathrm{Ar})\right]$, $121.6\left[\mathrm{~d}, J_{\mathrm{CP}}=4, \mathrm{C}^{3}(\mathrm{Ar})\right], 90.7(\mathrm{~s}, \mathrm{Cp}), 54.5,48.7,38.4,32.3,29.2$, 27.0, 24.2, $19.7\left(7 \times \mathrm{x} \mathrm{s}, \mathrm{CH}_{2}\right), 38.43\left[\mathrm{~s}, 2 \mathrm{x} \mathrm{C}^{1}\left({ }^{\mathrm{t}} \mathrm{Bu}\right)\right], 34.7$ [s, $\left.\mathrm{C}^{1}\left({ }^{\mathrm{t}} \mathrm{Bu}\right)\right]$, $34.3\left[\mathrm{~s}, 2 \times \mathrm{C}^{2}\left({ }^{\mathrm{t}} \mathrm{Bu}\right)\right], 30.9\left[\mathrm{~s}, \mathrm{C}^{2}\left({ }^{\mathrm{t}} \mathrm{Bu}\right)\right]$.

Preparation of $\left[\mathbf{M o C p}\left\{\mathbf{P}(\mathbf{O H}) \mathbf{R}^{*}\right\}(\mathbf{C O})_{2}\right]$ (2a). Neat $\mathrm{HBF}_{4} \cdot \mathrm{OEt}_{2}(4$ $\mu \mathrm{L}$ of a $54 \%$ solution in $\mathrm{Et}_{2} \mathrm{O}, 0.030 \mathrm{mmol}$ ) was added to a dichloromethane $(10 \mathrm{~mL})$ solution of compound $1(0.020 \mathrm{~g}, 0.03 \mathrm{mmol})$ to give instantaneously an orange solution. Solvent was then removed under vacuum, the residue was extracted with toluene and the extracts were filtered. Removal of the solvent from the filtrate gave an orange residue shown (by IR and ${ }^{31} \mathrm{P}$ NMR) to contain compound 2a as a major species. All attempts to further purify this crude material lead to its progressive deprotonation. $v_{\mathrm{CO}}\left(\mathrm{CH}_{2} \mathrm{Cl}_{2}\right): 1938$ (vs), 1858 (s) $\mathrm{cm}^{-1}$. ${ }^{31} \mathrm{P}\left\{{ }^{1} \mathrm{H}\right\} \mathrm{NMR}\left(\mathrm{CH}_{2} \mathrm{Cl}_{2}\right): \delta 336.6(\mathrm{~s})$.

Preparation of $\left[\mathrm{MoCp}\left\{\mathrm{P}(\mathrm{OMe}) \mathbf{R}^{*}\right\}(\mathrm{CO})_{2}\right] \quad$ (2b). A dichloromethane solution $(10 \mathrm{~mL})$ of compound $\mathbf{1}(0.030 \mathrm{~g}, 0.045$ mmol) was stirred at $0{ }^{\circ} \mathrm{C}$ with $\left[\mathrm{Me}_{3} \mathrm{O}\right] \mathrm{BF}_{4}(0.010 \mathrm{~g}, 0.067 \mathrm{mmol})$ for 2 min to give an orange solution. The solvent was then removed under vacuum, the residue was extracted with $\mathrm{CH}_{2} \mathrm{Cl}_{2}$ /petroleum ether (1:3) and the extracts chromatographed on alumina (activity IV) at $15^{\circ} \mathrm{C}$. Elution with the same solvent mixture gave an orange fraction. Removal of solvents from the latter gave compound $\mathbf{2 b}$ as an orange solid $(0.020 \mathrm{~g}, 84 \%)$. Anal. Calcd for $\mathrm{C}_{26} \mathrm{H}_{37} \mathrm{MoO}_{3} \mathrm{P}: \mathrm{C}, 59.54 ; \mathrm{H}, 7.11$. Found: C, 58.82; H, 6.91. $v_{\mathrm{CO}}\left(\mathrm{CH}_{2} \mathrm{Cl}_{2}\right): 1935$ (vs), $1856(\mathrm{~s}) \mathrm{cm}^{-1} .{ }^{1} \mathrm{H}$ NMR: $\delta 7.43\left(\mathrm{~d}, J_{\mathrm{HP}}=3,2 \mathrm{H}, \mathrm{C}_{6} \mathrm{H}_{2}\right), 5.14(\mathrm{~s}, 5 \mathrm{H}, \mathrm{Cp}), 3.98\left(\mathrm{~d}, J_{\mathrm{HP}}=14\right.$, $\left.3 \mathrm{H}, \mathrm{OCH}_{3}\right], 1.57\left(\mathrm{~s}, 18 \mathrm{H}, \mathrm{CH}_{3}\right), 1.32\left(\mathrm{~s}, 9 \mathrm{H}, \mathrm{CH}_{3}\right) .{ }^{31} \mathrm{P}\left\{{ }^{1} \mathrm{H}\right\} \mathrm{NMR}: \delta$ $350.5(\mathrm{~s}) .{ }^{13} \mathrm{C}\left\{{ }^{1} \mathrm{H}\right\}$ NMR: $\delta 238.5\left(\mathrm{~d}, J_{\mathrm{CP}}=20, \mathrm{CO}\right), 152.2\left[\mathrm{~s}, \mathrm{C}^{4}(\mathrm{Ar})\right]$, $150.2\left[\mathrm{~d}, J_{\mathrm{CP}}=6, \mathrm{C}^{2}(\mathrm{Ar})\right], 145.0\left[\mathrm{~d}, J_{\mathrm{CP}}=14, \mathrm{C}^{1}(\mathrm{Ar})\right], 123.3[\mathrm{~d}, J(\mathrm{CP})=$ 8, $\left.\mathrm{C}^{3}(\mathrm{Ar})\right], 93.0(\mathrm{~s}, \mathrm{Cp}), 55.7\left(\mathrm{~s}, \mathrm{OCH}_{3}\right), 39.0\left[\mathrm{~s}, 2 \times \mathrm{C}^{1}\left({ }^{\mathrm{t}} \mathrm{Bu}\right)\right], 35.3[\mathrm{~s}$, $\left.\mathrm{C}^{1}\left({ }^{\mathrm{t}} \mathrm{Bu}\right)\right], 34.3\left[\mathrm{~s}, 2 \times \mathrm{C}^{2}\left({ }^{\mathrm{t}} \mathrm{Bu}\right)\right], 31.1\left[\mathrm{~s}, \mathrm{C}^{2}\left({ }^{\mathrm{t}} \mathrm{Bu}\right)\right]$.

Preparation of $\left[\mathrm{MoCp}\left\{\mathbf{P}\left(\mathrm{O}_{2} \mathbf{C P h}\right) \mathbf{R} *\right\}(\mathbf{C O})_{2}\right] \quad$ (2c). A dichloromethane solution $(10 \mathrm{~mL})$ of compound $\mathbf{1}(0.036 \mathrm{~g}, 0.054$ mmol) was stirred with $\mathrm{PhC}(\mathrm{O}) \mathrm{Cl}(26 \mu \mathrm{L}, 0.22 \mathrm{mmol})$ for $2 \mathrm{~min}$. The solvent was then removed under vacuum to give a dark red residue which was extracted with petroleum ether. The extracts were filtered through alumina (activity IV, $2 \mathrm{~cm}$ ) and the solvent was then removed 
from the filtrate to give compound $2 \mathbf{c}$ as a purple solid $(0.026 \mathrm{~g}, 78 \%)$. The crystals used in the X-ray study were grown by slow diffusion at $20{ }^{\circ} \mathrm{C}$ of a layer of petroleum ether into a concentrated toluene solution of the complex. Anal. Calcd for $\mathrm{C}_{32} \mathrm{H}_{39} \mathrm{MoO}_{4} \mathrm{P}: \mathrm{C}, 62.54 ; \mathrm{H}, 6.40$. Found: C, 62.82; H, 6.58. $v_{\mathrm{CO}}\left(\mathrm{CH}_{2} \mathrm{Cl}_{2}\right): 1961$ (vs), 1882 (s), 1727 (w) $\mathrm{cm}^{-1} .{ }^{1} \mathrm{H}$ NMR: $\delta 8.07(\mathrm{~m}, 2 \mathrm{H}, \mathrm{Ph}), 7.61(\mathrm{~m}, 1 \mathrm{H}, \mathrm{Ph}), 7.49-7.44(\mathrm{~m}, 4 \mathrm{H}$, $\mathrm{Ph}$ and $\left.\mathrm{C}_{6} \mathrm{H}_{2}\right), 5.24(\mathrm{~s}, 5 \mathrm{H}, \mathrm{Cp}), 1.62\left(\mathrm{~s}, 18 \mathrm{H}, \mathrm{CH}_{3}\right), 1.35\left(\mathrm{~s}, 9 \mathrm{H}, \mathrm{CH}_{3}\right)$. ${ }^{31} \mathrm{P}\left\{{ }^{1} \mathrm{H}\right\}$ NMR: $\delta 290.6(\mathrm{~s}) .{ }^{13} \mathrm{C}\left\{{ }^{1} \mathrm{H}\right\}$ NMR: $\delta 237.9\left(\mathrm{~d}, J_{\mathrm{CP}}=23, \mathrm{CO}\right)$, $162.9\left[\mathrm{~d}, J_{\mathrm{CP}}=8, \mathrm{C}(\mathrm{O}) \mathrm{Ph}\right], 152.8\left[\mathrm{~s}, \mathrm{C}^{4}(\mathrm{Ar})\right], 150.7\left[\mathrm{~d}, J_{\mathrm{CP}}=5, \mathrm{C}^{2}(\mathrm{Ar})\right]$, $145.0\left[\mathrm{~d}, J_{\mathrm{CP}}=12, \mathrm{C}^{1}(\mathrm{Ar})\right], 133.8\left[\mathrm{~s}, \mathrm{C}^{4}(\mathrm{Ph})\right], 130.9$ [s, $\left.\mathrm{C}^{1}(\mathrm{Ph})\right], 130.5$ $\left[\mathrm{s}, \mathrm{C}^{2}(\mathrm{Ph})\right], 128.9\left[\mathrm{~s}, \mathrm{C}^{3}(\mathrm{Ph})\right], 123.5\left[\mathrm{~d}, J_{\mathrm{CP}}=9, \mathrm{C}^{3}(\mathrm{Ar})\right], 93.8(\mathrm{~s}, \mathrm{Cp})$, $39.2\left[\mathrm{~s}, 2 \times \mathrm{C}^{1}\left({ }^{\mathrm{t}} \mathrm{Bu}\right)\right], 35.4\left[\mathrm{~s}, \mathrm{C}^{1}\left({ }^{\mathrm{t}} \mathrm{Bu}\right)\right], 34.4\left[\mathrm{~s}, 2 \times \mathrm{C}^{2}\left({ }^{\mathrm{t}} \mathrm{Bu}\right)\right], 31.1[\mathrm{~s}$, $\left.\mathrm{C}^{2}\left({ }^{\mathrm{t}} \mathrm{Bu}\right)\right]$.

Preparation of $\left[\mathbf{M o C p}\left(\mathbf{P F R}^{*}\right)(\mathbf{C O})_{2}\right]$ (3). Neat $\mathrm{HBF}_{4} \cdot \mathrm{OEt}_{2}(12 \mu \mathrm{L}$ of a $54 \%$ solution in $\mathrm{Et}_{2} \mathrm{O}, 0.09 \mathrm{mmol}$ ) was added to a dichloromethane solution $(10 \mathrm{~mL})$ of compound 1 at $0{ }^{\circ} \mathrm{C}$ to give instantaneously an orange solution. Solvent was then removed under vacuum, the residue was extracted with petroleum ether and the extracts filtered. Solvent was then partially removed under vacuum from the filtrate and the concentrated solution was allowed to crystallize at $-20{ }^{\circ} \mathrm{C}$ to give compound 3 as X-ray quality red-purple crystals $(0.018 \mathrm{~g}, 80 \%)$. Anal. Calcd for $\mathrm{C}_{25} \mathrm{H}_{34} \mathrm{FMoO}_{2} \mathrm{P}: \mathrm{C}, 58.59 ; \mathrm{H}, 6.69$. Found: $\mathrm{C}, 58.11 ; \mathrm{H}, 6.52$. $v_{\mathrm{CO}}\left(\mathrm{CH}_{2} \mathrm{Cl}_{2}\right): 1959$ (vs), 1884 (s) $\mathrm{cm}^{-1} .{ }^{1} \mathrm{H}$ NMR (200.13 MHz): $\delta 7.45$ $\left(\mathrm{d}, J_{\mathrm{HP}}=3,2 \mathrm{H}, \mathrm{C}_{6} \mathrm{H}_{2}\right), 5.35(\mathrm{~s}, 5 \mathrm{H}, \mathrm{Cp}), 1.63\left(\mathrm{~s}, 18 \mathrm{H}, \mathrm{CH}_{3}\right), 1.32(\mathrm{~s}, 9 \mathrm{H}$, $\left.\mathrm{CH}_{3}\right) .{ }^{31} \mathrm{P}\left\{{ }^{1} \mathrm{H}\right\}$ NMR: $\delta 323.8\left(\mathrm{~d}, J_{\mathrm{PF}}=1132\right) .{ }^{13} \mathrm{C}\left\{{ }^{1} \mathrm{H}\right\}$ NMR: $\delta 236.0$ $\left(\mathrm{dd}, J_{\mathrm{CP}}=25, J_{\mathrm{CF}}=3, \mathrm{CO}\right), 153.6\left[\mathrm{~s}, \mathrm{C}^{4}(\mathrm{Ar})\right], 151.9\left[\mathrm{~d}, J_{\mathrm{CP}}=5, \mathrm{C}^{2}(\mathrm{Ar})\right]$, $144.9\left[\mathrm{~d}, J_{\mathrm{CP}}=24, \mathrm{C}^{1}(\mathrm{Ar})\right], 123.0\left[\mathrm{~d}, J_{\mathrm{CP}}=8, \mathrm{C}^{3}(\mathrm{Ar})\right], 94.0(\mathrm{~s}, \mathrm{Cp}), 38.4$ $\left[\mathrm{s}, 2 \times \mathrm{C}^{1}\left({ }^{\mathrm{t}} \mathrm{Bu}\right)\right], 35.5\left[\mathrm{~s}, \mathrm{C}^{1}\left({ }^{\mathrm{t}} \mathrm{Bu}\right)\right], 34.1\left[\mathrm{~s}, 2 \times \mathrm{C}^{2}\left({ }^{\mathrm{t}} \mathrm{Bu}\right)\right], 31.1[\mathrm{~s}$, $\left.\mathrm{C}^{2}\left({ }^{\mathrm{t}} \mathrm{Bu}\right)\right]$.

Preparation of $\left[\mathrm{MoCp}_{2}\left(\kappa^{2}-\mathrm{OPMeR}^{*}\right)(\mathbf{C O})_{2}\right]$ (4a). Neat $\mathrm{CH}_{3} \mathrm{I}(28$ $\mu \mathrm{L}, 0.45 \mathrm{mmol})$ was added to a dichloromethane solution $(10 \mathrm{~mL})$ of compound $1(0.030 \mathrm{~g}, 0.045 \mathrm{mmol})$ and the mixture was stirred at r.t. for $7 \mathrm{~h}$ to give a red solution. The solvent was then removed under vacuum, the residue extracted with dichloromethane/petroleum ether (1:6) and the extracts chromatographed on alumina (activity IV) at 15 ${ }^{\circ} \mathrm{C}$. A red fraction was collected using the same solvent mixture as eluent. Removal of solvents under vacuum from the latter fraction yielded compound $4 \mathbf{a}$ as a red solid $(0.022 \mathrm{~g}, 92 \%)$. Anal. Calcd for $\mathrm{C}_{26} \mathrm{H}_{37} \mathrm{MoO}_{3} \mathrm{P}: \mathrm{C}, 59.54 ; \mathrm{H}, 7.11$. Found: C, 58.60; H, 7.52. $v_{\mathrm{CO}}$ $\left(\mathrm{CH}_{2} \mathrm{Cl}_{2}\right): 1940$ (vs), 1850 (s). ${ }^{1} \mathrm{H}$ NMR: $\delta 7.37-7.33$ (m, ABX, $2 \mathrm{H}$, $\left.\mathrm{C}_{6} \mathrm{H}_{2}\right), 5.01(\mathrm{~s}, 5 \mathrm{H}, \mathrm{Cp}), 2.17$ [d, $\left.J_{\mathrm{HP}}=11,3 \mathrm{H}, \mathrm{PCH}_{3}\right), 1.64,1.53,1.31$ (3 x s, $\left.3 \times 9 \mathrm{H}, \mathrm{CH}_{3}\right) \cdot{ }^{31} \mathrm{P}\left\{{ }^{1} \mathrm{H}\right\}$ NMR: $\delta 28.3(\mathrm{~s}) .{ }^{13} \mathrm{C}\left\{{ }^{1} \mathrm{H}\right\}$ NMR: $\delta 255.8$ $\left(\mathrm{d}, J_{\mathrm{CP}}=26, \mathrm{CO}\right), 246.0(\mathrm{~s}, \mathrm{CO}), 157.3\left[\mathrm{~d}, J_{\mathrm{CP}}=18, \mathrm{C}^{2,6}(\mathrm{Ar})\right], 155.2[\mathrm{~s}$, $\left.\mathrm{C}^{4}(\mathrm{Ar})\right], 151.6\left[\mathrm{~s}, \mathrm{C}^{6,2}(\mathrm{Ar})\right], 132.9\left[\mathrm{~d}, J_{\mathrm{CP}}=34, \mathrm{C}^{1}(\mathrm{Ar})\right], 124.5$ [d, $J_{\mathrm{CP}}=$ 8, $\left.\mathrm{C}^{3,5}(\mathrm{Ar})\right], 123.1$ [d, $\left.J_{\mathrm{CP}}=14, \mathrm{C}^{5,3}(\mathrm{Ar})\right], 95.2(\mathrm{~s}, \mathrm{Cp}), 40.0,39.3,35.1$ $\left[3 \times \mathrm{x} \mathrm{s}, 3 \times \mathrm{C}^{1}\left({ }^{\mathrm{t}} \mathrm{Bu}\right)\right], 33.9,33.2,30.9\left[3 \times \mathrm{x} \mathrm{s}, 3 \times \mathrm{C}^{2}\left({ }^{\mathrm{t}} \mathrm{Bu}\right)\right], 23.8\left[\mathrm{~d}, J_{\mathrm{CP}}=\right.$ $\left.27, \mathrm{PCH}_{3}\right]$.

Preparation of $\left[\mathrm{MoCp}\left\{\kappa^{2}-\mathrm{OP}\left(\mathrm{C}_{3} \mathrm{H}_{5}\right) \mathrm{R}^{*}\right\}(\mathrm{CO})_{2}\right] \quad$ (4b). The procedure is identical to that described for $\mathbf{4 a}$, but using allyl bromide ( $48 \mu \mathrm{L}, 0.54 \mathrm{mmol}$ ) instead of MeI. After similar workup [elution with dichloromethane/petroleum ether (1:9)], compound $\mathbf{4 b}$ was obtained as a bright-red solid $(0.025 \mathrm{~g}, 84 \%)$. The crystals used in the X-ray study were grown by slow diffusion at $-20^{\circ} \mathrm{C}$ of a layer of petroleum ether into a concentrated toluene solution of the complex. Anal. Calcd for $\mathrm{C}_{28} \mathrm{H}_{39} \mathrm{MoO}_{3} \mathrm{P}: \mathrm{C}, 61.09 ; \mathrm{H}, 7.14$. Found: $\mathrm{C}, 61.52 ; \mathrm{H}, 7.42$. $v_{\mathrm{CO}}$ $\left(\mathrm{CH}_{2} \mathrm{Cl}_{2}\right): 1942$ (vs), 1852 (s). ${ }^{1} \mathrm{H}$ NMR: $\delta$ 7.39-7.35 (m, ABX, 2H, $\left.\mathrm{C}_{6} \mathrm{H}_{2}\right), 5.55(\mathrm{~m}, 1 \mathrm{H}, \mathrm{CH}), 5.15\left(\mathrm{~m}, 2 \mathrm{H}, \mathrm{CH}_{2}\right), 5.00(\mathrm{~s}, 5 \mathrm{H}, \mathrm{Cp}), 3.52(\mathrm{~m}$, $\left.2 \mathrm{H}, \mathrm{PCH}_{2}\right), 1.65,1.54,1.31\left(3 \mathrm{x} \mathrm{s}, 3 \times 9 \mathrm{H}, \mathrm{CH}_{3}\right) .{ }^{31} \mathrm{P}\left\{{ }^{1} \mathrm{H}\right\}$ NMR: $\delta 33.5$ (s). ${ }^{13} \mathrm{C}\left\{{ }^{1} \mathrm{H}\right\}$ NMR: $\delta 255.6\left(\mathrm{~d}, J_{\mathrm{CP}}=27, \mathrm{CO}\right), 246.5(\mathrm{~s}, \mathrm{CO}), 157.4[\mathrm{~d}$, $\left.J_{\mathrm{CP}}=19, \mathrm{C}^{2,6}(\mathrm{Ar})\right], 155.2\left[\mathrm{~s}, \mathrm{C}^{4}(\mathrm{Ar})\right], 151.6\left[\mathrm{~d}, J_{\mathrm{CP}}=4, \mathrm{C}^{6,2}(\mathrm{Ar})\right], 133.7$ $\left[\mathrm{d}, J_{\mathrm{CP}}=23, \mathrm{C}^{\mathrm{l}}(\mathrm{Ar})\right], 130.2\left(\mathrm{~d}, J_{\mathrm{CP}}=7, \mathrm{CH}\right), 124.6\left[\mathrm{~d}, J_{\mathrm{CP}}=8\right.$, $\left.\mathrm{C}^{3,5}(\mathrm{Ar})\right], 123.3\left[\mathrm{~d}, J_{\mathrm{CP}}=13 . \mathrm{C}^{5,3}(\mathrm{Ar})\right], 119.9\left(\mathrm{~d}, J_{\mathrm{CP}}=13, \mathrm{CH}_{2}\right), 95.1(\mathrm{~s}$, $\mathrm{Cp}), 40.3\left[\mathrm{~s}, \mathrm{C}^{1}\left({ }^{\mathrm{t}} \mathrm{Bu}\right)\right], 40.0\left(\mathrm{~d}, J_{\mathrm{CP}}=20, \mathrm{PCH}_{2}\right), 39.4\left[\mathrm{~d}, J_{\mathrm{CP}}=3\right.$, $\left.\left.\mathrm{C}^{1}\left({ }^{\mathrm{t}} \mathrm{Bu}\right)\right], 35.1\left[\mathrm{~s}, \mathrm{C}^{1}{ }^{1}{ }^{\mathrm{B} u} \mathrm{Bu}\right)\right], 34.1,33.4,31.0\left[3 \times \mathrm{x} \mathrm{s}, 3 \times \mathrm{C}^{2}\left({ }^{\mathrm{t}} \mathrm{Bu}\right)\right]$.
Preparation of $\left[\mathrm{MoCp}\left\{\mathrm{P}\left(\mathrm{OZrClCp}_{2}\right) \mathbf{R}^{*}\right\}(\mathrm{CO})_{2}\right]$ (5). Compound $\mathbf{1}$ $(0.042 \mathrm{~g}, 0.063 \mathrm{mmol})$ and $\left[\mathrm{ZrCl}_{2} \mathrm{Cp}_{2}\right](0.019 \mathrm{~g}, 0.065 \mathrm{mmol})$ were stirred in dichloromethane $(10 \mathrm{~mL})$ for $2 \mathrm{~min}$ to give a red solution. The solvent was then removed under vacuum, the residue extracted with toluene and the extracts filtered. Removal of solvent from the filtrate and washing of the residue with petroleum ether $(5 \mathrm{~mL})$ gave compound 5 as a red solid $(0.045 \mathrm{~g}, 92 \%)$. Satisfactory elemental analysis could not be obtained for this very water-sensitive product, as all attempts of crystallization resulted in its progressive hydrolysis. $v_{\mathrm{CO}}$ $\left(\mathrm{CH}_{2} \mathrm{Cl}_{2}\right): 1921$ (vs), 1841 (s). ${ }^{1} \mathrm{H}$ NMR: $\delta 7.41$ (d, $J_{\mathrm{HP}}=2,2 \mathrm{H}, \mathrm{C}_{6} \mathrm{H}_{2}$ ), $6.39(\mathrm{~s}, 10 \mathrm{H}, \mathrm{Cp}) 5.12(\mathrm{~s}, 5 \mathrm{H}, \mathrm{Cp}), 1.65\left(\mathrm{~s}, 18 \mathrm{H}, \mathrm{CH}_{3}\right), 1.31(\mathrm{~s}, 9 \mathrm{H}$, $\left.\mathrm{CH}_{3}\right) .{ }^{31} \mathrm{P}\left\{{ }^{1} \mathrm{H}\right\}$ NMR: $\delta 350.3$ (s).

Preparation of $\left[\mathrm{MoCp}\left\{\mathrm{P}(\mathrm{O}) \mathbf{R}^{*}\right\}(\mathrm{CO})_{2}\left(\mathrm{SnPh}_{3}\right)\right]$ (6). Compound 1 $(0.030 \mathrm{~g}, 0.045 \mathrm{mmol})$ and $\mathrm{SnPh}_{3} \mathrm{Cl}(0.037 \mathrm{~g}, 0.096 \mathrm{mmol})$ were stirred in dichloromethane for $2 \mathrm{~min}$ to give a yellow solution. Workup as described for 5 (filtration using a cannula) gave compound $\mathbf{6}$ as a yellow solid $(0.035 \mathrm{~g}, 90 \%)$. Satisfactory elemental analysis could not be obtained for this very water-sensitive product, as all attempts of crystallization resulted in its progressive hydrolysis. $v_{\mathrm{CO}}\left(\mathrm{CH}_{2} \mathrm{Cl}_{2}\right)$ : 1947 (s), 1889 (vs). ${ }^{1} \mathrm{H}$ NMR (200.13 MHz): $\delta 7.58-7.34$ (m, 17H, Ph and $\left.\mathrm{C}_{6} \mathrm{H}_{2}\right), 4.82(\mathrm{~s}, 5 \mathrm{H}, \mathrm{Cp}), 1.64\left(\mathrm{~s}, 18 \mathrm{H}, \mathrm{CH}_{3}\right), 1.35\left(\mathrm{~s}, 9 \mathrm{H}, \mathrm{CH}_{3}\right)$. ${ }^{31} \mathrm{P}\left\{{ }^{1} \mathrm{H}\right\}$ NMR $(81.03 \mathrm{MHz}): \delta 468.1\left(J_{119 \mathrm{SnP}}=J_{117 \mathrm{SnP}}=116\right)$.

$\mathrm{X}$-ray structure determination for compounds $1,2 \mathrm{c}, 3$ and $\mathbf{4 b}$. Crystallographic data for compounds $\mathbf{1}, \mathbf{2 c}, \mathbf{3}$ and $\mathbf{4 b}$ are presented respectively in the CIF file format. In each case a single crystal was coated in high-vacuum grease and mounted on a glass fibre. X-ray measurements were made using a Bruker SMART CCD area-detector diffractometer with Mo-K $\alpha$ radiation $(\lambda=0.71073 \AA){ }^{3}$ Intensities were integrated ${ }^{4}$ from several series of exposures, each exposure covering $0.3^{\circ}$ in $\omega$, and the total data set being a sphere $(\mathbf{4 b})$ or hemisphere $(\mathbf{1}, \mathbf{3}$ and $\mathbf{2 c}$ ). Absorption corrections were applied, based on multiple and symmetry-equivalent measurements using SADABS. ${ }^{5}$ The structures were solved by direct methods $(\mathbf{1}, \mathbf{3}$ and $\mathbf{2 c})$ or Patterson Synthesis $(\mathbf{4 b})$, and all were refined by least squares on weighted $\mathrm{F}^{2}$ values for all reflections. ${ }^{6}$ All non-hydrogen atoms were assigned anisotropic displacement parameters and refined without positional constraints. All hydrogen atoms were constrained to ideal geometries and refined with fixed isotropic displacement parameters. Hydrogen atom $\mathrm{H}(83)$ in compound 1 was located in the electron density difference map, assigned isotropic displacement parameters and refined without positional constraints. Complex neutral-atom scattering factors were used. ${ }^{7}$ Compound $\mathbf{4 b}$ crystallized with half a toluene molecule which was located at an inversion centre and was thus disordered in two inversion-related positions.

\section{References}

(1) D. D. Perrin, W. L. F. Armarego, Purification of Laboratory Chemicals. (Pergamon Press: Oxford, U.K., 1998).

(2) García, M. E.; Riera, V.; Ruiz, M. A.; Sáez, D.; Vaissermann, J.; Jeffery, J. C. J. Am. Chem. Soc., 2002, 124, 14304.

(3) SMART diffractometer control software, Bruker Analytical X-ray Instruments Inc., Madison, WI, 1998.

(4) SAINT integration software, Siemens Analytical X-ray Instruments Inc., Madison, WI, 1994.

(5) G. M. Sheldrick. SADABS: A program for absorption correction with the Siemens SMART system; University of Gottingen: Germany, 1996.

(6) SHELXTL program system version 5.1; Bruker Analytical X-ray Instruments Inc., Madison, WI, 1998.

(7) International Tables for Crystallography, Kluwer, Dordrecht, 1992, vol. C. 